\title{
INTENSIFICATION OF HEAT TRANSPORT FROM THE FURNACE TO HEAT ACCUMULATOR THROUGH A PHASE CHANGE
}

\begin{abstract}
In the article the authors analyse the process of heat transport from the furnace to a heat accumulator through a phase change of a heat carrier (working substance), design a construction and describe measurements done on experimental equipment. This type of heat transport is substantially more intense that the one in natural or forced convection and takes place without generating the working substance flow with a drive aggregate. Another advantage is heat accumulation control according to a pre-set temperature of an accumulator through an automatic flow control of the working substance in a condensation phase. The equipment consists of an autonomous evaporation section, a heat accumulator integrated with a condensation heat exchanger and of a thermostatically operated valve with thermal drive.
\end{abstract}

Keywords: Heat transport, LOOP heat pipe, heat accumulator.

\section{Introduction}

New constructions of external low thermal resistance building claddings reduce thermal losses, which can, in connection with excessive output of a fireplace liner, often lead to the overheating of rooms. Various flue gas heat and hot water exchangers are currently used to heat water for heating other rooms or for heat accumulation. Reduction of thermal radiation from fireplace liners can be also achieved by removing part of heat from the furnace and by its accumulation in some other place using the loop heat pipe. The loop heat pipe evaporator in a flat disk shape can be built in the furnace as one of its walls.

Efficient reduction of radiation heat from the furnace is to be provided by even cooling of the furnace radiating wall. A vertically situated evaporation area should have regular consumption; therefore, the uniform dispersion of fluid across the evaporation area is highly important. Its optimisation is substantial for efficient reduction of radiation heat from the furnace.

Water heating by means of a thermosyphon heat pipe enables to control transported heat output with a possibility of a complete shutdown of heat flow. Another advantage is that any freezingresistant medium can be used as a heat transferring substance. A hot water tank does not have to be placed in the immediate vicinity of the fireplace liner. This type of water heating can be widely used for low energy and passive houses where it can be used not only for water heating but also as an alternative heat source for houses equipped with gas or electric boilers. This system can also be used when there is no alternative source of energy.

\section{Haet pipes}

A heat pipe is a device for heat transport (heat output) while small temperature difference is maintained. This technology enables to transport large heat flows with devices of small dimensions. The device has no moving parts, is maintenance free and its service life is long. The heat pipe is a hermetically sealed pipe filled with a working substance, for example water, alcohol, freon, mercury, sodium, ammonia, helium, etc. at a defined pressure. The heating of one end of the pipe and the cooling of the other end cause the evaporation of the working medium on the heated end. Vapours flow through the pipe up to the cooled end where they condense again into liquid. The condensate flows either due to gravity or capillary elevation back to the warm end where it evaporates again. This gives rise to the working medium cycle connected with intense heat transport. Depending on how recovery flow of the condensate is provided, heat pipes are divided into: gravitational, rotary and capillary.

Figures 1 - 2 compare characteristics of a cylindrical copper rod of very good thermal conductivity $\left(\lambda_{\mathrm{Cu}}=380 \mathrm{~W} \cdot \mathrm{m}^{-1} \cdot \mathrm{K}^{-1}\right)$

\footnotetext{
* ${ }^{1}$ Milan Malcho, ${ }^{1}$ Stanislav Gavlas, ${ }^{2}$ Peter Hrabovsky, ${ }^{1}$ Roman Banovcan, ${ }^{1}$ Tomas Puchor, ${ }^{3}$ Lukasz Orman, ${ }^{4}$ Tadeas Ochodek ${ }^{1}$ Department of Power Engineering, Faculty of Mechanical Engineering, University of Zilina, Slovakia

${ }^{2}$ Research Centre, University of Zilina, Slovakia,

${ }^{3}$ Department of Piped Utility Systems, Faculty of Environmental, Geomatic and Energy Engineering, Kielce University of Technology, Poland

${ }^{4}$ Power Engineering Research Centre, VSB-TU Ostrava, Czech Republic

E-mail: milan.malcho@fstroj.uniza.sk
} 
of diameter $18 \mathrm{~mm}$ and length of $400 \mathrm{~mm}$ with those of a gravitational heat pipe made of a copper pipe of the same outside diameter with $1 \mathrm{~mm}$ wall thickness. In both cases the warm end was heated with hot water of ca. $73{ }^{\circ} \mathrm{C}$ and the cold end was cooled with cold water having $20^{\circ} \mathrm{C}$.

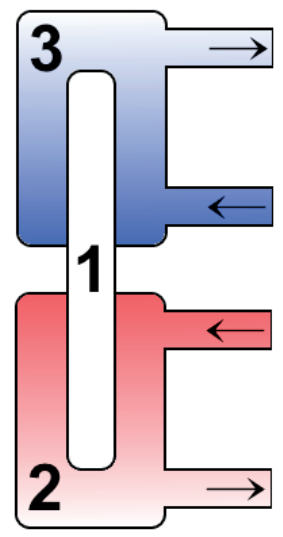

Fig. 1 Measurement of heat flow through GHP

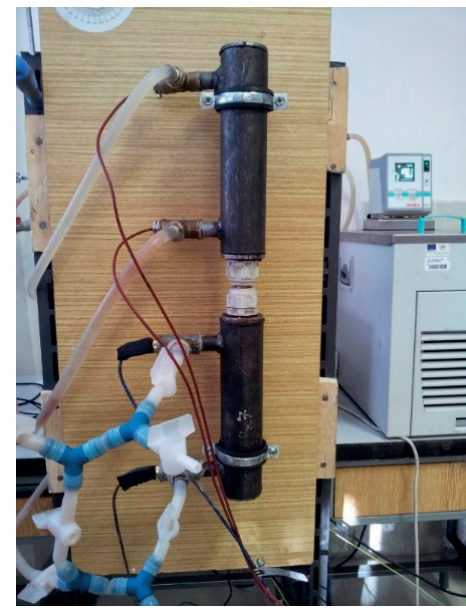

Fig. 2 Experimental device to measure heat flow through GHP with calorimetric method

It is obvious from Fig. 3 that the gravitational heat pipe with water as a working substance is able to transport through the circulating pump the heat output ten times greater than the copper rod at the same temperature gradient $\left(\mathrm{ca} .73^{\circ} \mathrm{C} / 20^{\circ} \mathrm{C}\right.$ ).

When there is an evaporator in the gravitational heat pipe placed below the condenser (in the direction of gravity), the capillary structure for condensate pumping can be omitted. Such an arrangement of the heat pipe is referred to as a thermosyphon loop.

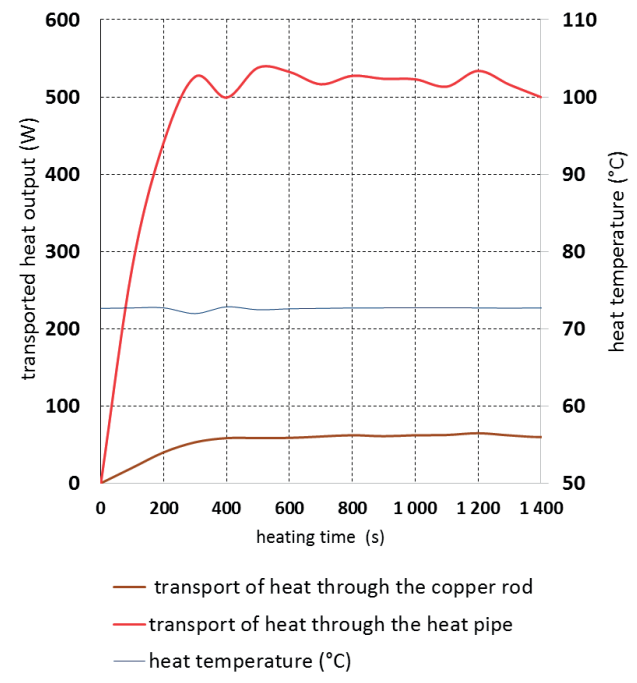

Fig. 3 Comparison of heat transport through a copper rod and heat pipe at a temperature of $73{ }^{\circ} \mathrm{C}$

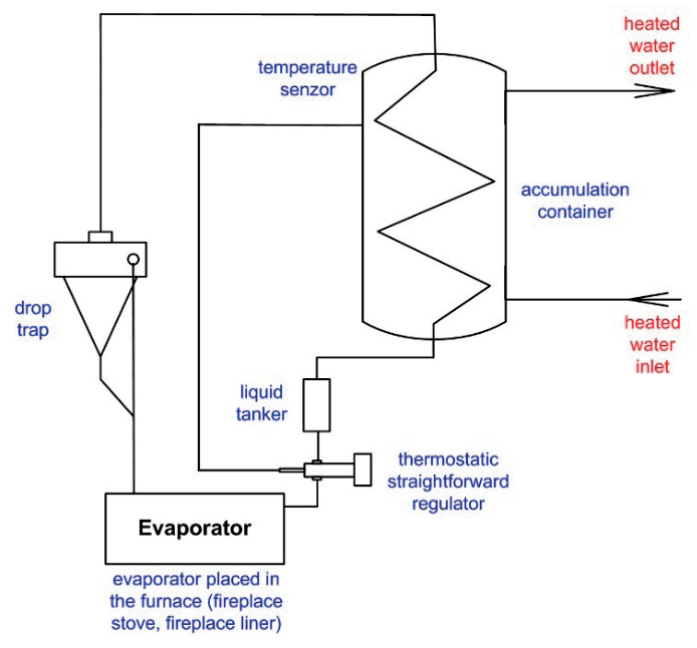

Fig. 4 Scheme of thermosyphon loop with control of transported heat output

The thermosyphon loop has been investigated by several authors [1]. Zohuri [2] published a study of the loop characteristics suitable for the cooling of a basic plate with power electronics and recommends suitable relationships for each component of heat transport and pressure loss in the system.

Figure 4 shows a thermosyphon loop in which in a steady state the fluid pressure in the condensate draining pipe must be equal to the pressure losses in the loop

$$
\begin{aligned}
\rho_{l} g h_{l}= & \Delta P_{\text {riser }}+\Delta P_{\text {condenser }}+ \\
& +\Delta P_{\text {downcomer }}+\Delta P_{\text {evaporator }}[P a],
\end{aligned}
$$

where $\rho_{l}$ is the liquid phase density, $\mathrm{g}$ is gravitational acceleration, $\mathrm{h}_{\mathrm{l}}$ is the height of the condensate column, $\Delta \mathrm{P}$ are pressure losses, riser, condenser, downcomer and evaporator. 
Determining the condenser pressure drop and downcomer we can assume that the working medium is only in a liquid phase Then,

$\Delta P_{\text {downcomer, condenser }}=\xi \frac{w^{2}}{2 \rho_{l}}$,

where $\xi$ is the empirical constant for the given geometry and $w$ is the velocity of condensate flow.

\section{Heat transport through the heat pipe}

Heat in the loop heat pipe is spread by radiation and convection from the furnace onto the evaporator wall. Further, it is spread by conduction and convection through a boiling liquid phase in the evaporator. Through a gaseous phase transport of the working substance flowing to the condenser and through its condensation it is transported onto the condenser wall. Heat flow continues by heat conduction and heat transfer from the exterior wall of the condenser to the accumulation container. The amount of heat output which the loop heat pipe can drain from the furnace to the accumulator container is mostly limited by the size of the furnace or by the size of a heat transfer surface from the side of the flame in the furnace.

Heat transport in a condensation heat exchanger (condenser) between a gaseous phase of the substance and an interior wall of the pipe is mainly influenced by the character of flows in the flowing liquid film of the condensate. The condensation section of the loop heat pipe is solved as a standard condensation heat exchanger with changing pressure which is dependent on the generating of a gaseous phase of the working substance in the evaporator. Heat transfer surfaces of the condensation heat exchanger and temperature of the accumulator filling are designed as to provide complete condensation of the working substance and cooling of the condensate in each mode of the furnace. Limits of heat output transported by a heat pipe in dependence on temperature are shown in Fig. 5.

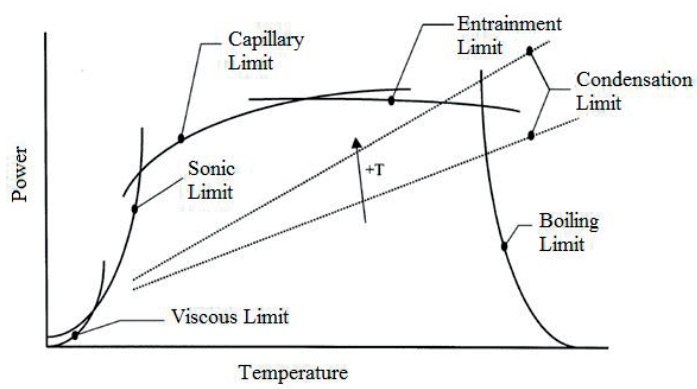

Fig. 5 Limits of heat output transported by a heat pipe in dependence on temperature [1]
Local density of heat flow in condensation of vapour on the condenser vertical wall in dependence on the distance of condensate layer occurrence $\mathrm{x}$ and on temperature difference on the condensate layer $\Delta \mathrm{t}_{\mathrm{i}, \mathrm{K}}$ is then

$q_{x}=\left(\frac{l_{v} \rho_{L} g \lambda_{l}^{3}}{4 v_{l} x}\right)^{0.25} \Delta t_{i, K}^{0.75}, \quad\left[W \cdot m^{-2}\right]$

where $l_{v}$ is condensation heat, $\lambda_{l}$ is thermal conductivity of the condensate, $v_{l}$ is kinematic viscosity of the condensate and $x$ is the distance of the place where the condensate is formed.

When huge amount of liquid boils and convection is natural, the coefficient of heat transfer depends on physical characteristics of liquid, pressure and temperature differences between the heating surface and liquid while the shape of the container does not play any role.

During heat transport in the evaporation section of the loop heat pipe, so called surface boiling or evaporation of the liquid layer is first observed. It occurs at lower heat flows and on the heated wall there are no large amounts of vapour bubbles [3].

Heat flow $\dot{Q}$, which is equal to a part of heat output generated by combustion of fuel in the furnace is conducted across the evaporator wall to the liquid.

Heat flow $\dot{Q}$ conducted across the evaporator wall is, according to Fourier relation, given by equation (4)

$\dot{Q}=q_{s p} \cdot S_{s p},[\mathrm{~W}]$

where $q_{s p}$ is the density of heat flow on the inner surface of the evaporator from the furnace direction which is expressed by relation (5)

$q_{s p}=\frac{\lambda_{v}}{\delta_{s}}\left(t_{s p}-t_{s v}\right),\left[\mathrm{W} \cdot \mathrm{m}^{-2} \cdot \mathrm{K}^{-1}\right]$

and $\delta_{S}$ is the real thickness of the evaporator wall.

Heat flow heats the liquid to its boiling point where the liquid state changes to a gaseous state. Heat flow is determined by Newton relation (6)

$\dot{Q}=\alpha_{v} \cdot S_{v} \cdot\left(t_{s v}-t_{2,3}\right),[\mathrm{W}]$

where $\alpha_{v}$ is the coefficient of heat transfer at liquid boiling, $\mathrm{S}_{\mathrm{v}}$ is the inner surface of the evaporator wall and $t_{\mathrm{sv}}$ is the temperature of the evaporator inner wall and $t_{2,3}$ is the temperature of saturated steam.

The heat transfer coefficient $\alpha_{v}$ can be determined from the Nusselt criterion according to relation (7)

$N_{u B}=\frac{\alpha_{v} \cdot L^{*}}{\lambda_{l}},[-]$ 
where $\lambda_{l}$ is the thermal conductivity of liquid and $\mathrm{L}^{*}$ is the characteristic dimension determined by relation (8)

$L^{*}=\frac{\sigma \cdot \rho_{l} \cdot c_{p} \cdot T_{2,3}}{\left(\rho_{v} \cdot l_{v}\right)},[\mathrm{m}]$

where $\sigma$ is the surface tension, $\rho_{1}$ is the condensate density, $c_{p}$ is the specific heat at constant pressure, $T_{2,3}$ is the absolute temperature of saturated steam, $\rho_{\mathrm{v}}$ is the density of vapour and 1 is evaporation heat.

The Nusselt criterion in the pressure range $1.105<\mathrm{p}<$ $170105 \mathrm{~Pa}$ is determined for bubble boiling of liquids in free convection according to relations (9) and (10)

$$
\begin{aligned}
& N_{u B}=0.0625 \operatorname{Re}_{B}^{0.5} \operatorname{Pr}_{K}^{0.333}, \text { for } 10^{-5} \leq \operatorname{Re}_{\mathrm{B}} \leq 10^{-2} \\
& N_{u B}=0.125 \operatorname{Re}_{B}^{0.65} \operatorname{Pr}_{K}^{0.333}, \text { for } 10^{-2} \leq \operatorname{Re}_{\mathrm{B}} \leq 10^{4}
\end{aligned}
$$

where $\mathrm{Re}_{\mathrm{B}}$ is the Reynolds criterion determined from relation (11), and $\operatorname{Pr}_{K}$ is the Prandtl criterion determined from relation (12)

$$
\operatorname{Re}_{B}=\frac{\omega_{v} \cdot L^{*}}{v_{l}},[-]
$$

$\operatorname{Pr}=\frac{v_{l}}{a_{l}},[-]$

where $\omega_{v}$ is the speed of vapour formation which is influenced by heat flow density as it results from relation (13). Vapour is then continually drained by the pipe from the evaporator to the condenser where it condenses and returns back in the form of a condensate. The speed of vapour formation can be determined according to the relation

$$
\omega_{v}=\frac{q_{v}}{\rho_{v} \cdot l_{v}},\left[\mathrm{~m} \cdot \mathrm{s}^{-1}\right]
$$

Temperature of the inner wall in the evaporator $t_{\mathrm{sv}}$ is then determined from relation (14), which results from relation (6)

$t_{s v}=\frac{q_{s p}}{\alpha_{v}}+t_{2,3}, \quad\left[{ }^{\circ} \mathrm{C}\right]$

And the temperature of the contact surface $t_{s p}$ is determined by simplification of relation (5) as

$\left.t_{s p}=\frac{q_{s p}}{\lambda_{v}}+t_{s v} .{ }^{\circ} \mathrm{C}\right]$

\section{Experimental device for measurement of heat transport through a phase change}

The main objective of the experiment was to verify the functionality of the device constructed for the accumulation of partial heat from the furnace of a local heat source to the heating of water based on the principle of heat transport through a phase change without the use of a circulating pump [4]. A component of such a device is the loop heat pipe which transports heat under optimal conditions (amount of filling, temperature) with high efficiency.

The device was designed with an aim to meet the requirements for amount of heat taken from combustion gases left from the fuel burnt in the furnace of a heat source. From the point of design and arrangement of individual components of the device it was necessary to locate the evaporation section of the heat pipe in the furnace so that combustion gases would be in contact with the surface of evaporation section which was directly irradiated with the flame. The device in Fig. 6 consists of the evaporator located in the back part of the combustion chamber of the heat source. The heat source is a double-ply fireplace liner with a rated output $11 \mathrm{~kW}$. The loop heat pipe evaporator is built in the back top part of the combustion chamber (Fig. 7). It is made of $4 \mathrm{~mm}$ carbon steel plates suitable for hot water and vapour boilers. Outer dimensions are 500×200×20 mm. To avoid deformation of the evaporator walls which might be caused by the increased pressure at reduced heat consumption, the inner space is rib-reinforced. On the back evaporator wall there are DN 15 steel tubes diagonally placed to supply and remove the working substance in gaseous and liquid state. The hot water container or accumulator container is in the vicinity of the heat source because of the compactness and possibility of immediate checking of individual parts of the heat device. The container is of a cylindrical shape and its volume is 110 litres. The accumulation container is made from stainless steel material AISI 304. In the top section of the container there is a welded flange for checking and exchanging the condensation section of the heat pipe. The condenser of the loop heat pipe is in a shape of a helical spiral made from an $18 \mathrm{~mm}$ copper pipe which is fastened inside the container with vacuum tight screws. Heat is transported from the furnace on the basis of the phase change from liquid to gas and vice versa. Heat transport is initiated with the processes of boiling and condensation which take place in the evaporator and accumulation container at the existing difference between the temperature of vapour and the temperature of hot water in the accumulation container. Sources of heat can be various types of energies, for example, natural gas, propane butane, wood, wood briquettes. Heat transport from the combustion chamber to the accumulator container in this experimental device is carried out by the thermosyphon circulation without forced circulation of the working substance in the loop. 


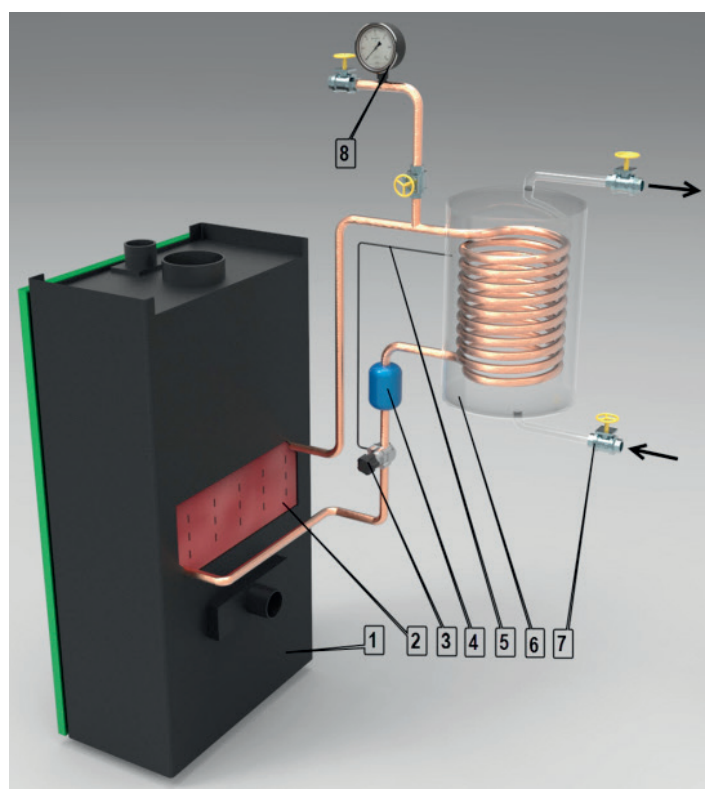

Fig. 6 Connection of a heat accumulator and a condensation heat exchanger to the furnace back wall of the fireplace liner: 1. Fireplace stove, 2. Evaporator, 3. Thermostatic valve, 4. Reservoir, 5. Thermodrive,

6. Heat accumulator, 7. Cold water supply, 8. Pressure gange
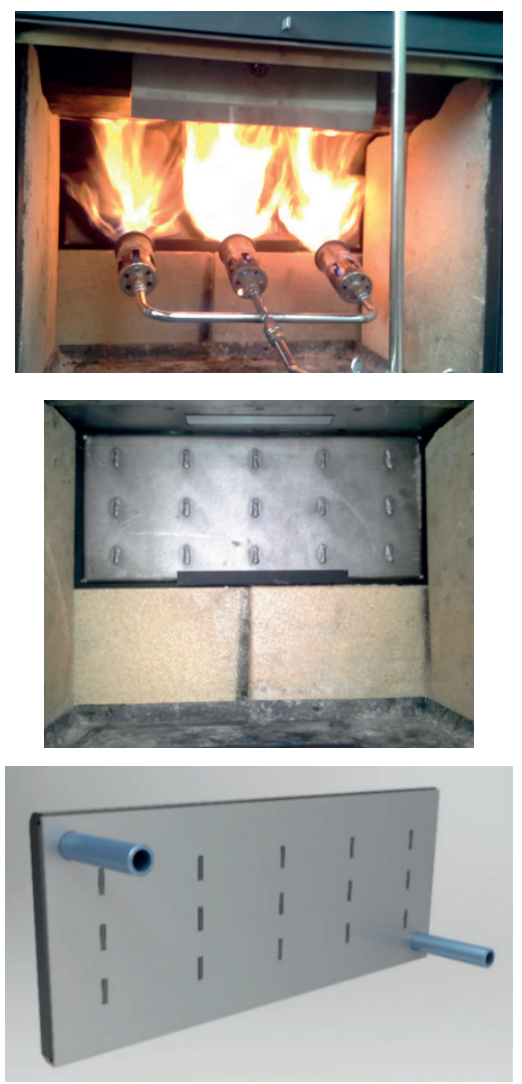

Fig. 7 Evaporator of the loop gravitational heat pipe and its location in the furnace
The pipe transporting the substance (either in a gaseous or liquid state) is thermally isolated and is considered to be an adiabatic part of the heat pipe (the transport of heat from the source to the destination without any heat losses) - Fig. 8. It connects the input and output of the evaporation and condensation part and is made from a copper pipe with an outside diameter $18 \mathrm{~mm}$. The transport pipe contains also a condensate trap and reservoir of the working substance. The condensate trap connects the evaporation pipe with the condensation pipe and its task is to drain the condensate pushed out with vapour back to the evaporation section. To separate the liquid phase from the gaseous phase there is a cylindrical metal grid with $0.1 \mathrm{~mm}$ openings placed in the condenser trap. Behind the condenser trap only the gaseous phase of the working substance flows; it continues further to the condensation section and the separated liquid phase flows through the pipe from the drop trap back to the evaporation section of the loop heat pipe. The working substance reservoir is placed next to the hot water container and traps the condensate after the required temperature of hot water has been reached. It forms a kind of reserve space which was not included in the heat pipe volume when filling volume of the working substance was designed. After the required temperature of hot water in the accumulation container is reached, the reservoir is filled with the liquid working substance so that the condensate surface in the condenser achieves the maximum height of the connection to the condenser after the condensate flow to the evaporator is stopped with the thermostatic valve. After hot water is taken from the accumulation container, the inlet of cold water cools the liquid thermometer of the thermoregulatory valve and, consequently, the thermoregulatory valve on the condensate inlet to the heat pipe evaporator is opened. The condensate begins to flow to the evaporator and heat begins to be transported from the evaporator to the accumulator. On the condensate pipe there are apart from the thermoregulatory valve also a discharge valve and an impulse flow meter for the measurement of condensate volume. The thermoregulatory valve automatically regulates the heat pipe output on the basis of the chosen and pre-set temperature of hot water in the accumulation container. The impulse flow meter placed behind the condensation section of the loop heat pipe enables to determine also instantaneous heat output of the device through mass flow of the condensate drained from the heat exchanger in the accumulation container. For the assessment of vacuum tightness of the loop heat pipe system and measurement of absolute pressure there is an absolute pressure sensor placed in the vapour section of the pipe [5]. Thermocouple sensors $\mathrm{NiCr}-\mathrm{Ni}$ and resistance sensors Pt100 were used for measurement of individual temperatures; they read input and output temperatures from the evaporator and condenser and temperature profile in the accumulation container. 


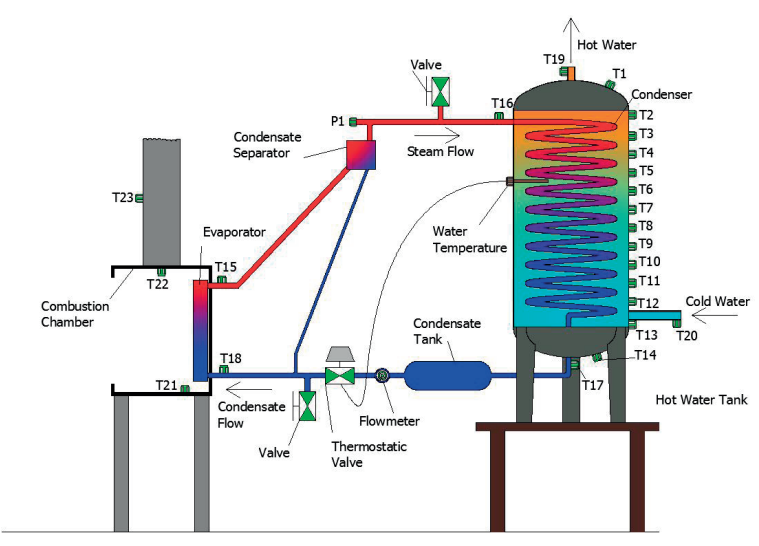

Fig. 8 Scheme of experimental device

(T1 - T23 Temperature sensors, P1 pressure sensor)

\section{Realisation and results of experiments}

In experiments focusing on verification of the operation of the device providing heat transport from the furnace to the heat accumulator through a phase change two fuels were used. In the first experiment propane butane with combustion heat 50.35 MJ.kg ${ }^{-1}$ was used (Fig. 9) as an energy carrier. When gas burners were used, water heating was initiated in the accumulator with water filling. The thermoregulatory valve is fully open and the condensate flow at hot water heating in the accumulator is maximal. Flow measurement shown in models the situation when water in the condenser is heated to the pre-set temperature (ca. $40{ }^{\circ} \mathrm{C}$ ), the thermostat stops the condensate flow and shuts of the heating. Then the consumption of hot water begins. Within this consumption period, the temperature of water decreases and the thermostat again opens the condensate flow to the evaporator. Hot water consumption will be stopped, utility water will be again heated to the original temperature and the water heating will be stopped again. The measurement also shows the situation after the second closing of the thermostat.

Its repeated opening was recorded at the temperature of $36^{\circ} \mathrm{C}$, which represents the switching difference of $4^{\circ} \mathrm{C}$. From this point of view it is a comfort operating mode, which should be suitable for most consumers. The repeated heating of water to the original temperature lasted 25 minutes and the average output of the heat pipe at that time was $5.7 \mathrm{~kW}$.

When testing the behaviour of the system for heat transport from the furnace to the heat accumulator using a loop heat pipe also dry lump wood was used as fuel. An objective of the measurement was not to achieve a maximum output but to observe the behaviour of the loop heat pipe in a real situation. The output initiation when wood (Fig. 10) is used is not as fast as when gas burners are used. The flame is not focused directly to the exchanger area as in the case when measurement is performed with gas burners. The achieved outputs are lower, their courses are not so steady and they have a tendency to oscillate. This can be seen mostly during after flaming. The oscillation is caused by shortage of heat supplied to the evaporator. After it is filled with a cooled condensate, it takes some time while the working
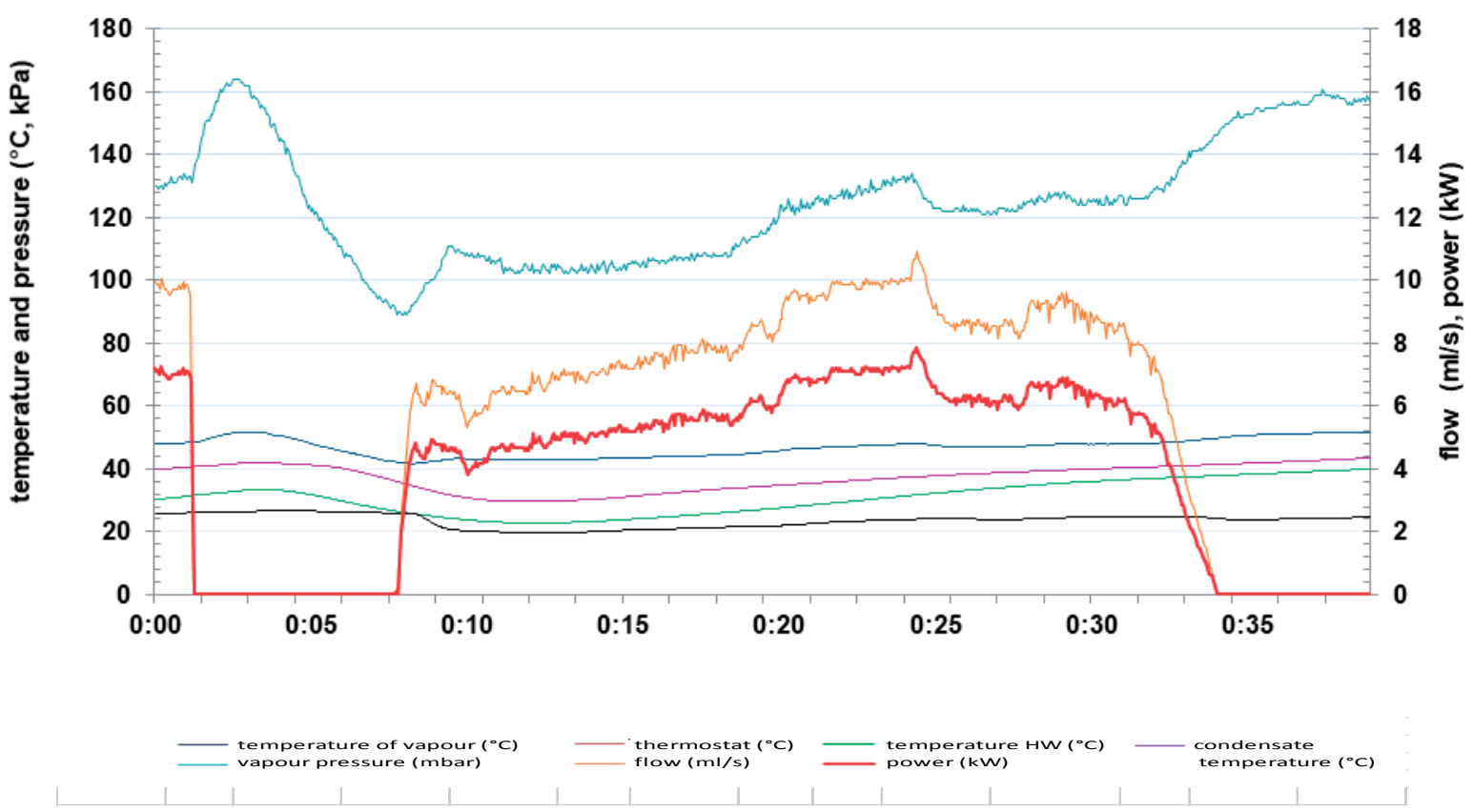

Fig. 9 Courses of temperatures, absolute pressure of vapour phase and transported heat output through a loop HP to the accumulation container with propane butane as a fuel 

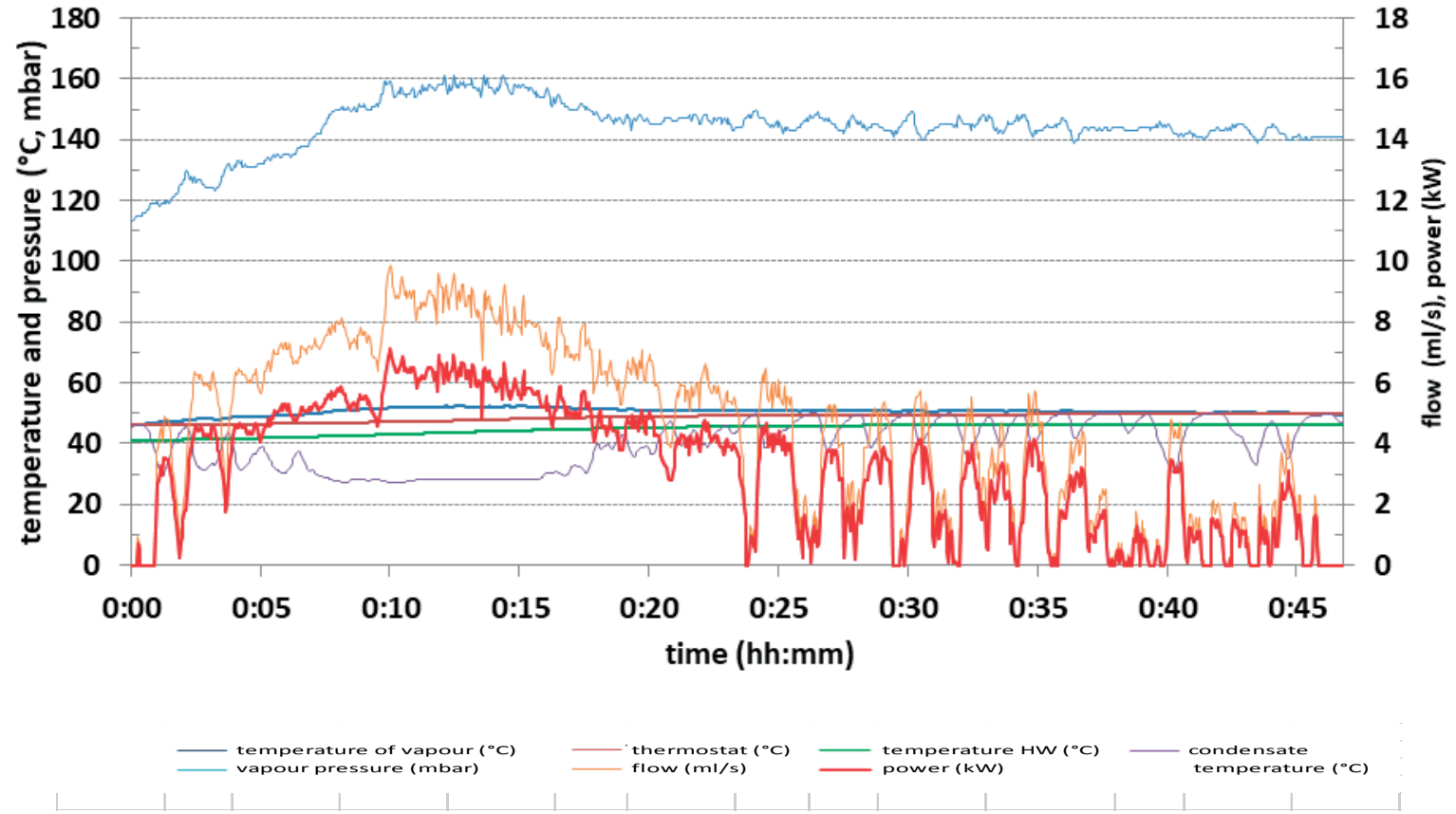

Fig. 10 Output and temperature parameters of the loop heat pipe when burning beech wood

substance is heated and evaporates. The maximum value of transmitted output calculated from the condensate flow achieved $7 \mathrm{~kW}$.

\section{Conclusion}

The device for transport of part of heat output from the furnace of the local heat source to the heat accumulator through a phase change meets the requirements for safe and long-term operation without any other auxiliary energy for circulation of the working medium or for regulation of its flow.

The proposed heat circuit can be implemented in different furnaces or in other heat sources of hot combustion gases as, for example, diesel and other engines and industrial furnaces.
Water heating by means of the thermosyphon heat pipe brings the regulation of transported heat output with a possibility of a complete shutdown of heat flow. Another advantage is that any freezing-resistant medium can be used as a heat transferring substance. The hot water container does not have to be placed in the immediate vicinity of a heat source. The suggested way of water heating can be widely used also for low-energy and passive houses in which various forms of renewable energy sources are used.

\section{Acknowledgment}

This work was solved within the project APVV-15-0778 "Limits of radiation and conventional cooling through phase changes of the working substance in the loop thermosyphon.

\section{References}

[1] REAY, D. A, KEW, P. A.: Heat Pipes, Theory, Design and Applications, $5^{\text {th }}$ ed., Burlington, 377 p., MA 01803, 2006.

[2] ZOHURI, B.: Heat Pipe Design and Technology, Taylor and Francis Group, 462 p., FL 33487-2742, 2011.

[3] RADEK, N., ORMAN, L. J.: Water Boiling on Electro-Spark Deposited Copper Coating, ВICHИК, No. 6, 2008, 233-236, ISSN 2226-9150.

[4] MALCHO, M., JANDACKA, J., GAVLAS, S.: Device for Automatic Transport of Heat with Self Adjustable Temperature of Water (in Slovak), 5042-2014, 29.09.2014

[5] NEMEC, P., MALCHO, M., SMITKA, M., MATUSOV, J.: Performance Parameters of Closed Loop Thermosyphon, Communications - Scientific Letters of the University of Zilina, vol. 14, No. 4a, 2012, 53-57, ISSN 1335-4205. 